\title{
Response to treatment in patients with pulmonary tuberculosis in Central Plateau of Iran during 2012- 2018
}

\author{
Jamshid Ayatollahi ${ }^{1}$, Mohammad Sharifyazdi ${ }^{1}$, Masoud Hafizi ${ }^{2}$, Masoomeh Shirvani ${ }^{3 *}$, Seyed \\ Hossein Shahcheraghi ${ }^{{ }^{*}}$
}

1 Infectious Diseases Research Center, Shahid Sadoughi Hospital, Shahid Sadoughi University of Medical Sciences, Yazd, Iran

2 Infectious \& Tropical Diseases Specialist, Department of Infectious \& Tropical Diseases, Faculty of Medicine, Shahrekord University of Medical Sciences, Shahrekord, Iran

3 Medical Student, Shahid Sadoughi University of Medical Sciences, Yazd, Iran

*Corresponding Author:

Masoomeh Shirvani, Medical Student, Shahid Sadoughi University of Medical Sciences, Yazd, Iran.

Tel: +98-3538229200, E-mail: masoomsh.1370@gmail.com

Dr. Seyed Hossein Shahcheraghi, Infectious Diseases Research Center, Shahid Sadoughi University of Medical Sciences, Yazd, Iran.

Tel: +98-9132531389, E-mail: shahcheraghih@gmail.com

Received: September 01, 2019; Accepted: October 02, 2019

\begin{abstract}
Tuberculosis is one of the outstanding infectious diseases and one of the most vital reasons for mortality around the world. The prevalence and spread of pulmonary tuberculosis and the consequences of the failure of the treatment of this disease, determining the success rate of treatment among the patient with tuberculosis and looking at the quantity of treatment disappointments is of high significance. In this study, the response of patients with TB to the treatment in Chaharmahal and Bakhtiari province in 2012-2018 has been evaluated. A descriptive cross-sectional study has been conducted. All the information about patients referring to this center on the basis of the questionnaire during the years 2012 to 2018 has been reviewed. For this study, there were 165 patients with pulmonary and extra pulmonary tuberculosis. Of the 111 patients with pulmonary tuberculosis, 11 cases $(9.9 \%)$ died, all of whom died albeit for the problems other than tuberculosis. In data newest related to 2018,21 cases of pulmonary tuberculosis were diagnosed, of which 12 cases (10.8\%) were being under treatment and thus the result of their treatment has yet to be determined. Out of 86 patients with pulmonary tuberculosis, 83 cases $(96.5 \%)$ were successful and 3 cases $(3.5 \%)$ had failure. It can be inferred that the exact implementation of the DOTS strategy as well as the low rate of non-Iranian nationalities in Chaharmahal and Bakhtiari province are among those important factors that made the successful treatment in patient with pulmonary tuberculosis there.
\end{abstract}

Keywords: Pulmonary tuberculosis, Treatment, Iran.

\section{INTRODUCTION}

Tuberculosis (TB) is a medical problem with a yearly frequency rate of about 8.6 million cases and an estimated 1.3 million deaths every year. It still positions among the main ten reasons for death around the world [1]. TB can cause infection in persons with impaired immunity [2]. Most infections do not have symptoms, known as latent TB, where in about $10 \%$ of these latent infections can create sickness in lifetime which can kill about half of those infected [3]. The symptoms of active pulmonary TB disease are coughing, with sputum or blood, chest pains, fever, weight loss and night sweats [4]. TB is transmitted through beads from a tainted individual with dynamic aspiratory ailment discharged in the air through cough, sneeze or talking and then inhaled by another person [5]. TB can also spread via ingesting infected meat or milk (bovine TB) [6].

Over $60 \%$ of the global TB burden are related to Africa and Asia, 
where India, Indonesia and China account for the most rate of cases amounting to $43 \%$ of the world burden [7].

Medical assessment of TB incorporates therapeutic history of introduction, contamination and other hazard factors like HIV disease; physical examination to evaluate patient's general wellbeing to educate treatment plan; chest x-ray to detect chest abnormalities; and microbiological tests using samples of sputum [4].

According to a recent investigation by the World Health Organization (WHO) in 2016, almost 10 million from population were infected with Mycobacterium tuberculosis and 1.7 million died (including .4 million deaths among HIV)-positive cases) due to TB [7].

By and large, The treatment results are affected by sociodemographic and socio-economic factors [8]; nutrition [9]; HIV [7]; MDR TB [2, 3]; and strategies for TB administration including DOTS.

The standard TB treatment regimen comprises of an escalated stage that lasts 2 months involving 4 drugs (isoniazid, rifampicin, pyrazinamide and ethambutol) to quickly execute the Mycobacterium tuberculosis; and a continuation stage that last up to 4 months including 2 drugs (isoniazid, rifampicin) that take out the rest of the bacilli and avert backslide [10].

It has been discovered that poor adherence and unpredictable TB treatment prompts an expansion in the time of disease with the outcomes of multi-drug resistance TB (MDR-TB) [11, 12].

Investigation of treatment outcome of newly diagnosed smear positive pulmonary tuberculosis (PTB) patients is used as a main pointer to gauge the effectiveness of national tuberculosis program (NTP) [13].

WHO recommended Directly Observed Treatment Short course (DOTS) [14, 15], which goes for distinguishing $70 \%$ of irresistible cases and restoring $85 \%$ of them. This procedure is relied upon to interfere with transmission of the malady and lessen the quantity of contaminated people and the time of infectiousness [12].

It is an important subject to investigate very more about treatment outcome of TB patients in Iran. Therefore, this study was conducted to investigate this problem in the central area of Iran.

\section{METHODOLOGY}

In this descriptive cross-sectional study, all tuberculosis cases between 2012 and 2018 were studied. Our target community in this study was all patients with tuberculosis who referred to the health centers of Chaharmahal and Bakhtiari province during the mentioned years.

Sampling method was as simple census. Sample size included all patients with pulmonary tuberculosis who referred to health centers of Chaharmahal and Bakhtiari province during the last six years and were diagnosed and reported to the health center of Chaharmahal and Bakhtiari province.

In order to collect the required information, a questionnaire was prepared in which age, sex, nationality, place of involvement of tuberculosis (pulmonary and extra pulmonary), smear of onset of treatment, smear of the end of the second month, smear of the end of the third month, smear of the end of fourth and fifth months, the cultivation of the end of the third month, the cases of relapse, the patients 'deaths and the patients' treatment status (success of treatment, failure of treatment, transfer to other centers, absence from treatment, treatment, mistake in diagnosis) has been documented. Subsequently, referring to the health center of Chaharmahal and Bakhtiari province, the information was extracted from the files related to tuberculosis patients during this period.

The research limitations included items related to ESR testing of patients, drug addiction, HIV infection, drug side effects, and patient information deficiencies.

\section{RESULTS}

For this study, there were 165 patients with pulmonary and extra pulmonary tuberculosis. Of these, 54 cases (32.7\%) had extrapulmonary tuberculosis and 111 cases $(67.3 \%)$ had pulmonary tuberculosis. Our goal is to study pulmonary tuberculosis.

Of the 111 cases of pulmonary TB, 63 cases $(56.8 \%)$ were men and 48 cases $(43.2 \%)$ were women. As regard the treatment status of patients, 83 case $(74.8 \%)$ had succeeded treatment and 3 cases $(2.7 \%)$ had failed treatment and 2 cases $(1.8 \%)$ had transmitted to other centers. Of the 111 patients with tuberculosis, only one of them had non-Iranian nationalities and has transmitted to other centers. Of the 111 patients with pulmonary tuberculosis, 11 cases $(9.9 \%)$ died, all of whom died albeit for the problems other than tuberculosis. In 2018, 21 cases of pulmonary tuberculosis were diagnosed, of which 12 cases $(10.8 \%)$ were being under treatment and thus the result of their treatment has yet to be determined.

Considering the fact that some of patients with pulmonary tuberculosis are being treated and their treatment status is unclear and also, given that all deaths have been for the reasons other than tuberculosis, 86 cases of patients whose treatment status were well-defined, have been reviewed in terms of the treatment status. Thus, out of 86 patients with pulmonary tuberculosis, 83 cases $(96.5 \%)$ were successful and 3 cases $(3.5 \%)$ had failure.

To study the treatment status of the patients in terms of their age, they have been divided in four age groups (Table 1).

Studying the result of treatment of patients in terms of their sex showed that 44 cases $(95.6 \%)$ of the men had successful treatment and 2 cases (4.4\%) had failed treatment, and 39 cases $(97.5 \%)$ of the women had successful treatment, and 1 case $(2.5 \%)$ had failed treatment.

The results of treatment of patients based on positive sputum smear and negative sputum smear were evaluated. Out of 62 cases of positive sputum smear, 60 cases (96.8\%) had successful treatment and 2 cases (3.2\%) had failed treatment and, out of 24 cases of negative sputum smear, 23 cases $(95.8 \%)$ were successful and 1 case $(4.2 \%)$ was failure to treatment.

The lowest incidence of tuberculosis in the year $2013(8.1 \%)$ and the highest incidence rate was in 2015 (21.6\%) (Table 2).

Because of low number of reported failure cases, evaluation of $\mathrm{p}$-value in order to determine the relationship between response to treatment of tuberculosis and age, gender and positive or 
negative sputum smear were not possible.

Table 1: The frequency of the treatment status of the TB patients based on age groups

\begin{tabular}{|l|l|c|c|c|c|}
\hline \multicolumn{2}{|c|}{ Age group } & $<30$ & $30-50$ & $51-70$ & $>70$ \\
\multirow{2}{*}{ Status of treatment } & & & & \\
\hline \multirow{2}{*}{ Successful } & $\mathrm{N}$ & 14 & 25 & 26 & 18 \\
\cline { 2 - 7 } & $\%$ & 93.3 & 96.1 & 96.3 & 100 \\
\hline \multirow{2}{*}{ Failure } & $\mathrm{N}$ & 1 & 1 & 1 & 0 \\
\cline { 2 - 7 } & $\%$ & 6.7 & 3.9 & 3.7 & 18 \\
\hline \multirow{2}{*}{ Total } & $\mathrm{N}$ & 15 & 26 & 27 & 100 \\
\cline { 2 - 7 } & $\%$ & 100 & 100 & 100 & 0 \\
\hline
\end{tabular}

Table 2: Incidence of tuberculosis in the patients with pulmonary tuberculosis based on year

\begin{tabular}{|c|c|c|}
\hline Year & N & $\%$ \\
\hline 2012 & 14 & 12.7 \\
\hline 2013 & 9 & 8.1 \\
\hline 2014 & 22 & 19.8 \\
\hline 2015 & 24 & 21.6 \\
\hline 2016 & 21 & 18.9 \\
\hline $2017 \&$ start of 2018 & 21 & 100 \\
\hline Total & 111 & \\
\hline
\end{tabular}

\section{DISCUSSION}

In this study, 54 cases $(32.7 \%)$ had extra-pulmonary tuberculosis and 111 cases (67.3\%) had pulmonary tuberculosis. 44 cases $(95.6 \%)$ of the men had successful treatment and 2 cases (4.4\%) had failed treatment, and 39 cases $(97.5 \%)$ of the women had successful treatment, and 1 case $(2.5 \%)$ had failed treatment. Also, out of all cases of positive sputum smear, 60 cases $(96.8 \%)$ had successful treatment and 2 cases $(3.2 \%)$ had failed treatment and, out of 24 cases of negative sputum smear, 23 cases $(95.8 \%)$ were successful and 1 case $(4.2 \%)$ was failure to treatment.

A study evaluated TB treatment outcome among new smear positive TB patients in Malaysia. Out of 110 cases in unsuccessful outcome program, 30 defaulted from the treatment, 59 died and 21 were transferred to other health centers. Results showed that the treatment success rate among the new smear positive TB patients was $67.26 \%$ that is less than the success aim set by WHO [1]. In the present study, we had $96.8 \%$ successful treatment among cases of positive sputum smear.
In another study by Atif $\mathrm{M}$, et al treatment success rate of $67.8 \%$ among new and retreatment TB patients and 69\% in new smear positive TB patients was investigated. The success rate for treatment among TB cases was lower than the recorded $85 \%$ success rate [16]. Therefore, we had a good research in the present study with $96.8 \%$ successful treatment toward mentioned study.

Hamusse SD, et al also investigated this subject in Ethiopia. The treatment success rate varied from 69.3 to $92.5 \%$, defaulter rate from 2.5 to $21.6 \%$, death rate from 1.6 to $11.1 \%$, and failure rate from 0 to $3.6 \%$ across the 25 districts of the zone [17]. Increasing of treatment success in this study was similar to present study.

The outcome of another study in Taiwan was classified as cured in $46(27.7 \%)$, treatment completed in $73(44.0 \%)$, died in 27 $(16.3 \%)$, treatment failed in five $(3.0 \%)$, and defaulted in 15 $(9.0 \%)$ [18]. 


\section{CONCLUSION}

Our results indicated that the treatment success rate among the smear positive TB patients was $96.8 \%$. This rate can be increased by monitoring the treatment progress of the patients with a greater number of cases in several areas of Iran.

\section{Acknowledgement}

The authors wish to thank the staff of Infectious Diseases Research Center of Shahid Sadoughi University of Medical Sciences, Yazd.

\section{Conflict of Interest}

We declare that we have no conflict of interest.

\section{Financial Support}

None declared.

\section{REFERENCES}

1. Atif M, Sulaiman SAS, Shafie AA, Ali I, Asif M. Treatment outcome of new smear positive pulmonary tuberculosis patients in Penang, Malaysia. BMC infectious diseases 2014;14(1):399.

2. Narasimhan P, Wood J, Maclntyre CR, Mathai D. Risk factors for tuberculosis. Pulmonary medicine 2013;2013.

3. Esmail H, Barry C, Young D, Wilkinson R. The ongoing challenge of latent tuberculosis. Phil Trans $R$ Soc $B$ 2014;369(1645):20130437.

4. Khalif AM, Karanja S, Mohammed K. Factors associated with tuberculosis treatment outcomes among tuberculosis patients attending tuberculosis treatment centres in 2016-2017 in Mogadishu, Somalia. The Pan African Medical Journal 2017;28.

5. Qian H, Li Y, Nielsen P, Hyldgaard C, Wong T, Chwang A. Erratum: Dispersion of exhaled droplet nuclei in a two-bed hospital ward with three different ventilation systems (Indoor Air (2006) 16,(111-128)). Indoor Air. 2006.

6. Tschopp R, Schelling E, Hattendorf J, Aseffa A, Zinsstag J. Risk factors of bovine tuberculosis in cattle in rural livestock production systems of Ethiopia. Preventive veterinary medicine 2009;89(34):205-11.

7. Raviglione M, Sulis G. Tuberculosis 2015: burden, challenges and strategy for control and elimination. Infectious disease reports 2016;8(2).

8. Vesosky B, Turner J. The influence of age on immunity to infection with Mycobacterium tuberculosis. Immunological reviews 2005;205(1):229-43.

9. Mehta JB, Emery MW, Girish M, Byrd JR, Roy TM. Atypical Pott's disease: localized infection of the thoracic spine due to Mycobacterium avium-intracellulare in a patient without human immunodeficiency virus infection. Southern medical journal 2003;96(7):685-8.

10. Sia IG, Wieland ML, editors. Current concepts in the management of tuberculosis. Mayo Clinic Proceedings; 2011: Elsevier.

11. Lönnroth K, Migliori GB, Abubakar I, D'Ambrosio L, de Vries G, Diel $R$, et al. Towards tuberculosis elimination: an action framework for low-incidence countries. European Respiratory Journal 2015;45(4):928-52.

12. Migliori GB, Sotgiu G, Lange C, Centis R. Extensively drugresistant tuberculosis: back to the future. Eur Respiratory Soc 2010.

13. Helbling P, Medinger C, Altpeter E, Raeber P-A, Beeli D,
Zellweger J-P. Outcome of treatment of pulmonary tuberculosis in Switzerland in 1996. Swiss Medical Weekly 2002;132(35/36):51722.

14. Yassin MA, Datiko D, Shargie E. Ten-year experiences of the tuberculosis control programme in the southern region of Ethiopia. The International Journal of Tuberculosis and Lung Disease 2006;10(10):1166-71.

15. Keshavjee S, Farmer PE. Tuberculosis, drug resistance, and the history of modern medicine. New England Journal of Medicine 2012;367(10):931-6.

16. Atif M, Anwar Z, Fatima RK, Malik I, Asghar S, Scahill S. Analysis of tuberculosis treatment outcomes among pulmonary tuberculosis patients in Bahawalpur, Pakistan. BMC research notes 2018;11(1):370.

17. Hamusse SD, Demissie M, Teshome D, Lindtjørn B. Fifteen-year trend in treatment outcomes among patients with pulmonary smear-positive tuberculosis and its determinants in Arsi Zone, Central Ethiopia. Global health action 2014;7(1):25382.

18. Lee J-J, Wu R-L, Lee Y-S, Wu Y-C, Chiang C-Y. Treatment outcome of pulmonary tuberculosis in eastern Taiwanexperience at a medical center. Journal of the Formosan Medical Association 2007;106(1):25-30. 